\title{
Possible contribution of nitric oxide and prostaglandin in the protective effect of angiotensin (1-7) against stress induced gastric ulceration in adult male albino rats
}

\author{
Hassan MKA ${ }^{1}$, Aziz NM${ }^{1}$, Shaaban $\mathrm{MAE}^{2}$, Rifaai RA ${ }^{3}$ \\ Department of Physiology, Faculty of Medicine, Minia University, Minia, Egypt. emadmax71@yahoo.com
}

\begin{abstract}
To assess the gastro-protective potential of the angiotensin (Ang-) (1-7) on the gastric secretion and ulceration induced by cold restraint stress (CRS) in adult male rats and the possible contribution of nitric oxide and prostaglandin $\mathrm{E}_{2}$. Rats were pylorically ligated and divided randomly into the following groups (8 rats each): control, cold-restraint stressed (CRS), stressed Ang-(1-7) treated, stressed L-NNA-Ang-(1-7) treated, stressed Indo-Ang-(1-7) treated groups. Our results revealed that Ang-(1-7) pre-treatment proved to be protective against development of ulcerative lesions in CRS model as evidenced by histological examination and the reduction of the ulcer index and this could be mediated through reduction of free and total acidity and pepsin concentration of gastric secretion with significantly decreased lipid peroxidation and increased the gastric protective nitric oxide and prostaglandin $\mathrm{E}_{2}$ levels. Furthermore, Ang-(1-7) pre-treatment has anti-apoptotic effect, evident by its down-regulation of the CRS induced over-expression of the gastric caspase 3 . In addition, the gastro-protective effects of Ang-(1-7) were significantly attenuated by co-administration with L-NNA or indomethacin. In conclusion, Ang-(1-7) can be considered a potential therapeutic agent to protect against the major clinical challenge of gastric injury resulting from stress. Nitric oxide and prostaglandin $E_{2}$ seem to contribute to the Ang-(1-7)'s gastro-protective effect (Tab. 2, Fig. 5, Ref. 35). Text in PDF www.elis.sk. KEY WORDS: angiotensin (1-7), cold restraint stress, Nitric oxide, prostaglandin $\mathrm{E}_{2}$, caspase 3.
\end{abstract}

\section{Introduction}

The circulating Renin-angiotensin system (RAS) is a wellrecognized hormonal system, which plays an important role in control of the cardiovascular system and extracellular fluid volume. More recently, it has been recognized that local RAS is present in most organs, including the gastrointestinal tract. The components of local RAS are present in the stomach, small intestine, colon, pancreatic islets, and liver (1).

The Angiotensin (1-7) is an important component of the RAS (2). Ang-(1-7) is a downstream peptide generated from angiotensin I through three known enzymes neprilysin (NEP), thimetoligopeptidase (TOP), and prolyloligopeptidase (POP)). Ang. I is quickly degraded to Ang-(1-7) in the rat stomach and the formation of Ang-(1-7) may even precede Ang. I conversion to Ang. II (3).

Gastrointestinal tract is one of the areas, where the role of nitric oxide (NO) is studied and results are controversial. In the

${ }^{1}$ Department of Physiology, Faculty of Medicine, Minia University, Minia, Egypt, ${ }^{2}$ Department of Physiology, Faculty of Medicine, AL-Azhar University, Assuit, Egypt, and ${ }^{3}$ Department of Histology, Faculty of Medicine, Minia University, Minia, Eygpt

Address for correspondence: Neven Makram Aziz, Department of Physiology, Faculty of Medicine, Minia University, 61111, Minia, Egypt Phone: +2.0122256894 , Fax: +2.086 .2324414$ gastrointestinal tract (GIT), NO participates in the modulation of the smooth musculature tone, such as the regulation of intestinal peristalsis, gastric emptying and antral motor activity. It also regulates acid and gastric mucus secretion, alkaline production and is involved in the maintenance of mucosal blood flow (4). On the other hand, gastric mucosal integrity requires the continuous generation of prostaglandin $\mathrm{E}_{2}\left(\mathrm{PGE}_{2}\right)$, which is crucial for the protection against ulcerogenic and necrotizing agents (5).

Gastric epithelium is often attacked by physical, chemical or microbiological agents acting from the gastric lumen. Among the numerous injurious luminal agents and irritants of both exogenous and endogenous origin, the stomach is a site of massive production and concentration of reactive oxygen species (ROS), far higher than other tissues or biological fluids. ROS provoke severe changes at the cellular level leading to cell death because of their extreme reactivity (6).

There is not a single cause for peptic ulcer, but the exact aetiology is the presence of imbalance between aggressive factors (e.g., acidity, pepsin, inflammation and oxidative stress) and defensive factors (e.g., mucus, bicarbonate and GMB flow) (7). So, the present work was a trial to evaluate the role of Ang-(1-7) in the pathophysiologic mechanisms of experimentally induced gastric ulceration caused by cold-restraint stressed (CRS). In addition, the possible involvements of nitric oxide and prostaglandins as targets for the ulcer-protective action of Ang-(1-7) were investigated by 
testing the effect of exogenous Ang-(1-7) against stress ulcerogenesis in the presence of nitric oxide synthase (NOS) inhibitor, $\mathrm{N} \omega$-nitro-L-Arginine (L-NNA) and non-selective cyclo-oxygenase (COX) inhibitors (Indomethacin).

\section{Materials and methods}

\section{Animals}

Forty adult male albino (Sprague dawley strain) rats, of average weight $150-180 \mathrm{gm}$, about 4 months old, were used in the present study. Rats were purchased from the National Research Centre, Cairo, Egypt. All rats were housed in groups of eight in stainless steel cages that offered adequate space for free movement and wandering ( $40 \mathrm{~cm}$ x $40 \mathrm{~cm}$ x $25 \mathrm{~cm}$ ). They were fed with commercial rat chow and left freely wandering in their cage for two weeks with normal hour's dark: light cycle for acclimatization before starting the experiment. All the experimental procedures were in accordance with our institutional guidelines. The ethics of the protocol were approved by The Laboratory Animals' Maintenance and Usage Committee of Faculty of Medicine, Minia University.

\section{Induction of gastric ulceration}

At the end of the experiment, rats were deprived of food for $24 \mathrm{~h}$ prior to the experiment in mesh-bottomed cages to minimize coprophagia, but allowed free access to water (8). Pyloric ligation was carried out under light ether anaesthesia according to the previous method described by Alumets et al (9). After pyloric ligation, the animals were immediately restrained by fixing the four limbs to a wooden board and placed in a refrigerator at $4{ }^{\circ} \mathrm{C}$ for 3 hours to induce cold-restraint stressed (CRS) ulcer (10). All experiments were performed at the same time of the day to avoid variations due to diurnal rhythms of putative regulators of gastric functions.

\section{Experimental procedures}

Rats were randomly divided into the following groups (8 rats each):
1) Control group $(C)$; in which rats received no treatment and were left freely wandering in their cages for 3 hours after being subjected to pyloric ligation.

2) CRS group; in which rats received no treatment before exposure to CRS.

3) Stressed Ang-(1-7) treated group; in which rats were treated by Ang-(1-7) (6.5 mg/kg, i.p) (Sigma, USA) (11) one hour before exposure to CRS.

4) Stressed L-NNA-Ang-(1-7) treated group; in which the rats were treated by Ang-(1-7) $(6.5 \mathrm{mg} / \mathrm{kg}$, i.p.) thirty minutes after being treated by L-NNA (20 mg/kg, i.p) (Sigma, USA) (12) and after another thirty minutes rats were exposed to CRS.

5) Stressed Indo-Ang-(1-7) treated group; in which the rats were treated by Ang-(1-7) $(6.5 \mathrm{mg} / \mathrm{kg}$, i.p) thirty minutes after being treated by indomethacin ( $5 \mathrm{mg} / \mathrm{kg} / \mathrm{i} . \mathrm{p}$ ) (Sigma, USA) (13) and after another thirty minutes rats were exposed to CRS.

Three hours after pyloric ligation, the rats were anesthetized by light ether anaesthesia, and blood samples were taken from the jugular vein. Then their stomachs were removed, opened along the greater curvature and the gastric content of each stomach was collected. The stomachs were washed with ice-cold saline and examined for gross gastric mucosal lesions using a magnified lens by an observer not aware of the experiment.

\section{Assessment of gastric mucosal lesions}

The gastric mucosal lesions were expressed in the form of ulcer index (U.I.) according to the method of Robert et al (14) and the preventive index of a given drug was calculated according the method previously described by Hano et al (15).

\section{Analysis of the gastric juice}

The gastric juice collected after opening the stomachs was centrifuged at $1000 \mathrm{~g}$ for 10 minutes to remove any solid debris and the volume of the supernatant was measured. The supernatant was then analysed for determination of $\mathrm{pH}$ according to the method of Hara et al (16), free and total acid concentrations were estimated

Tab. 1. Effect of the cold restraint stress with or without treatment on the gastric juice parameters in pyloric ligated rats.

\begin{tabular}{|c|c|c|c|c|c|}
\hline \multirow{2}{*}{ Parameters } & \multicolumn{5}{|c|}{ Groups } \\
\hline & Control & CRS & Stressed Ang- (1-7) & Stressed L-NNA-Ang- (1-7) & Stressed Indo-Ang- (1-7) \\
\hline Volume (ml/3 h) & $2.5 \pm 0.1$ & $1.4 \pm 0.1^{\mathrm{a}}$ & $2.4 \pm 0.1^{\mathrm{b}}$ & $1.9 \pm 0.02^{\mathrm{abc}}$ & $1.8 \pm 0.1^{\mathrm{abc}}$ \\
\hline $\mathrm{pH}$ & $3.4 \pm 0.2$ & $1.2 \pm 0.2^{\mathrm{a}}$ & $3.2 \pm 0.05^{\mathrm{b}}$ & $2.8 \pm 0.1^{\mathrm{abc}}$ & $2.6 \pm 0.1^{\mathrm{abc}}$ \\
\hline F.A.C. $(\mathrm{mEq} / \mathrm{L})$ & $17.5 \pm 1.1$ & $68.4 \pm 2.5^{\mathrm{a}}$ & $20.5 \pm 1.4^{b}$ & $46.8 \pm 1.4^{\mathrm{abc}}$ & $49.3 \pm 1.5^{\mathrm{abc}}$ \\
\hline T.A.C. $(\mathrm{mEq} / \mathrm{L})$ & $36.5 \pm 1.2$ & $88.4 \pm 2.1^{\mathrm{a}}$ & $40.5 \pm 1.5^{\mathrm{b}}$ & $63.8 \pm 1.8^{\mathrm{abc}}$ & $68.3 \pm 1.7^{\mathrm{abc}}$ \\
\hline Pepsin $(\mu \mathrm{g} / \mathrm{ml}$ tyrosin $)$ & $138.7 \pm 2.3$ & $225 \pm 5.4^{\mathrm{a}}$ & $143.4 \pm 2.1^{\mathrm{b}}$ & $162.2 \pm 2.2^{\mathrm{abc}}$ & $165 \pm 2.1^{\mathrm{abc}}$ \\
\hline
\end{tabular}

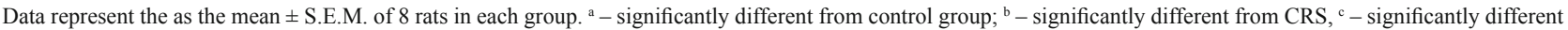
from Ang-(1-7) treated group, $\mathrm{p} \leq$ 0.05. F.A.C.: Free Acid Concentration; T.A.C.: Total Acid Concentration.

Tab. 2. Effect of the cold restraint stress with or without treatment on ulcer profile in pyloric ligated rats.

\begin{tabular}{|c|c|c|c|c|c|}
\hline Groups & $\%$ incidence & M.S.S. & M.U.S. & U.I. & P.I. (\%) \\
\hline Control & 0 & 0 & 0 & 0 & 0 \\
\hline CRS & 100 & 0.5 & 2.0 & 12.5 & - \\
\hline Stressed Ang-(1-7) & 16 & 0.1 & 0.16 & 1.86 & 85.1 \\
\hline Stressed L-NNA-Ang-(1-7) treated & 33 & 0.31 & 0.6 & 4.21 & 66.3 \\
\hline Stressed Indo-Ang-(1-7) treated & 35 & 0.36 & 0.8 & 4.66 & 63.3 \\
\hline
\end{tabular}

M.S.S. - Mean Severity Score, M.U.S. - Mean Ulcer Score, U.I. - Ulcer Index, P.I. - Preventive Index 
by using the method of Pratha et al (17) and pepsin concentrations were determined by a modified spectrophotometric method (18).

\section{Biochemical analysis of gastric mucosa}

The stomach of each rat was divided into the two parts: one part was immersed in IND $(10 \mu \mathrm{g} / \mathrm{ml})$ for 20 minutes to inhibit further formation and release of PGs and then stored at $-80{ }^{\circ} \mathrm{C}$. Subsequently, the gastric mucosa was scraped, homogenized in 2 $\mathrm{ml}$ normal saline containing $0.1 \mathrm{M}$ dithiothreitol and centrifuged at $2000 \mathrm{~g}$ for 10 minutes at room temperature. The supernatant was analysed for determination of prostaglandin content $\left(\mathrm{PGE}_{2}\right)$ by enzyme-linked immunosorbent assay (ELISA) using $\mathrm{PGE}_{2}$ assay kit (R\&D Systems, USA). The other part of gastric mucosa was also scraped, homogenized in cold potassium phosphate buffer ( $0.05 \mathrm{M}, \mathrm{pH} 7.4)$ and centrifuged at $2000 \mathrm{~g}$ for 10 minutes at $4{ }^{\circ} \mathrm{C}$. The supernatant was kept at $-80^{\circ} \mathrm{C}$ for subsequent measurement of lipid peroxides and nitric oxide using colorimetric assay kit according to the recommendations of the manufacturer (Biodiagnostic, Egypt).

\section{Histological examination}

Specimens were fixed in $10 \%$ neutral-buffered formalin, dehydrated, cleared, and embedded in paraffin wax. Tissue sections of 5-6 $\mu \mathrm{m}$ thickness were obtained and deparaffinised. Some sections were stained with hematoxylin and eosin (19). For immunohistochemical detection of Caspase 3 expression, Ultra Vision ONE HRP polymer detection system (Thermo Fisher Scientific Inc./Lab Vision, Fremont, CA, USA) was used according to the manufacturer's protocol. The slides were then counterstained, dehydrated, and mounted (20).

\section{Statistical analysis}

Data were represented as means \pm standard errors of the mean (SEM). Statistical analysis was performed using Graph pad Prism 5 software and significant difference between groups was done by one-way ANOVA followed by Tukey-Kramar post hoc test for multiple comparisons with a value of $\mathrm{p} \leq 0.05$ considered statistically significant.

\section{Results}

- The results clearly demonstrated that exposure of the rats to CRS significantly lowered the volume and $\mathrm{pH}$ of gastric juice, which was accompanied by a significantly higher gastric juice free and total acidity and the pepsin activity as compared to the control rats (Tab. 1). In addition, there was a significant higher level of gastric mucosal lipid peroxidase, with lower levels of $\mathrm{NO}$ and $\mathrm{PGE}_{2}$ as compared to the control group (Figs 1, 2, 3). Subjecting the rats to CRS was also associated with a high ulcer index reaching 12.5 (Tab. 2).

- Pre-treatment of CRS rats with Ang-(1-7) proved to be protective against development of ulcerative lesions as evidenced by the decrease in the ulcer index to 1.86 and achieving preventive index $85.1 \%$ (Tab. 2). In addition, Ang-(1-7) pre-treatment showed lower levels of gastric juice free and total acidity and the pepsin

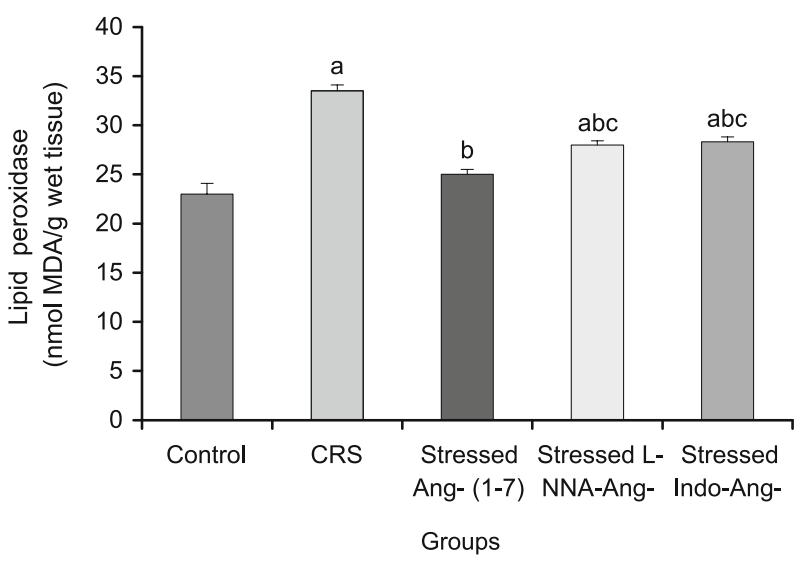

Fig. 1. Lipid peroxidase level in the different studied groups. Data represent the as the mean \pm S.E.M. of 8 rats in each group. ${ }^{a}-$ significantly different from control group; ${ }^{b}$ - significantly different from CRS, ${ }^{\mathrm{c}}$ - significantly different from Ang-(1-7) treated group, $\mathrm{p} \leq \mathbf{0 . 0 5}$.

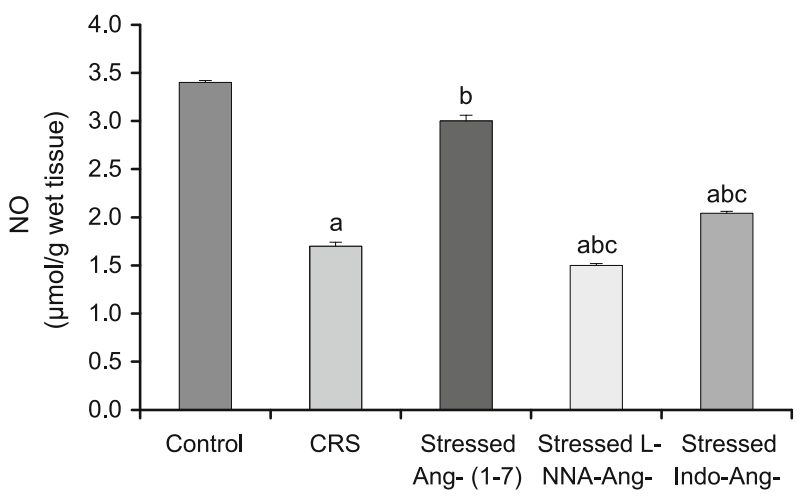

Fig. 2. Nitric oxide (NO) level in the different studied groups. Data represent the as the mean \pm S.E.M. of 8 rats in each group. ${ }^{a}$ - significantly different from control group; ${ }^{\mathrm{b}}$ - significantly different from CRS, ${ }^{\mathrm{c}}$ - significantly different from Ang-(1-7) treated group, $\mathrm{p} \leq \mathbf{0 . 0 5}$.

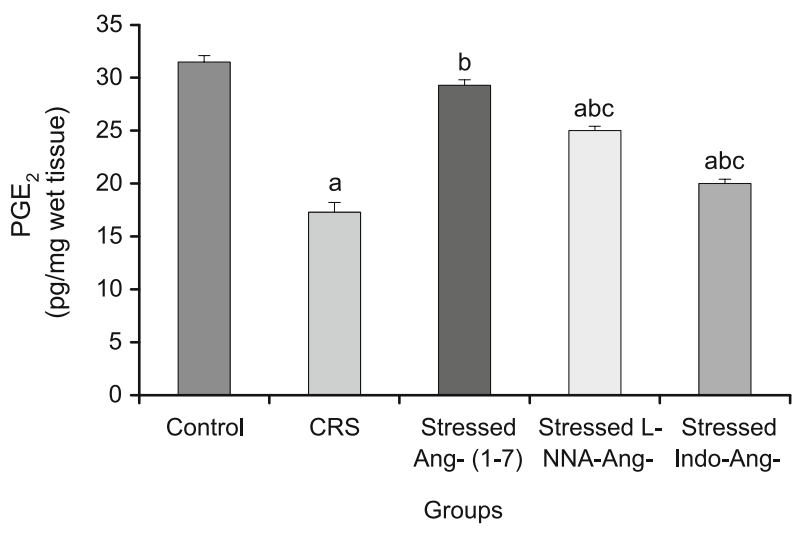

Fig. 3. Prostaglandin $\mathrm{E}_{2}\left(\mathrm{PGE}_{2}\right)$ level in the different studied groups. Data represent the as the mean \pm S.E.M. of 8 rats in each group. ${ }^{a}$ - significantly different from control group; ${ }^{\mathrm{b}}$ - significantly different from CRS, ${ }^{\mathrm{c}}$ - significantly different from Ang-(1-7) treated group, $\mathrm{p} \leq \mathbf{0 . 0 5}$. 

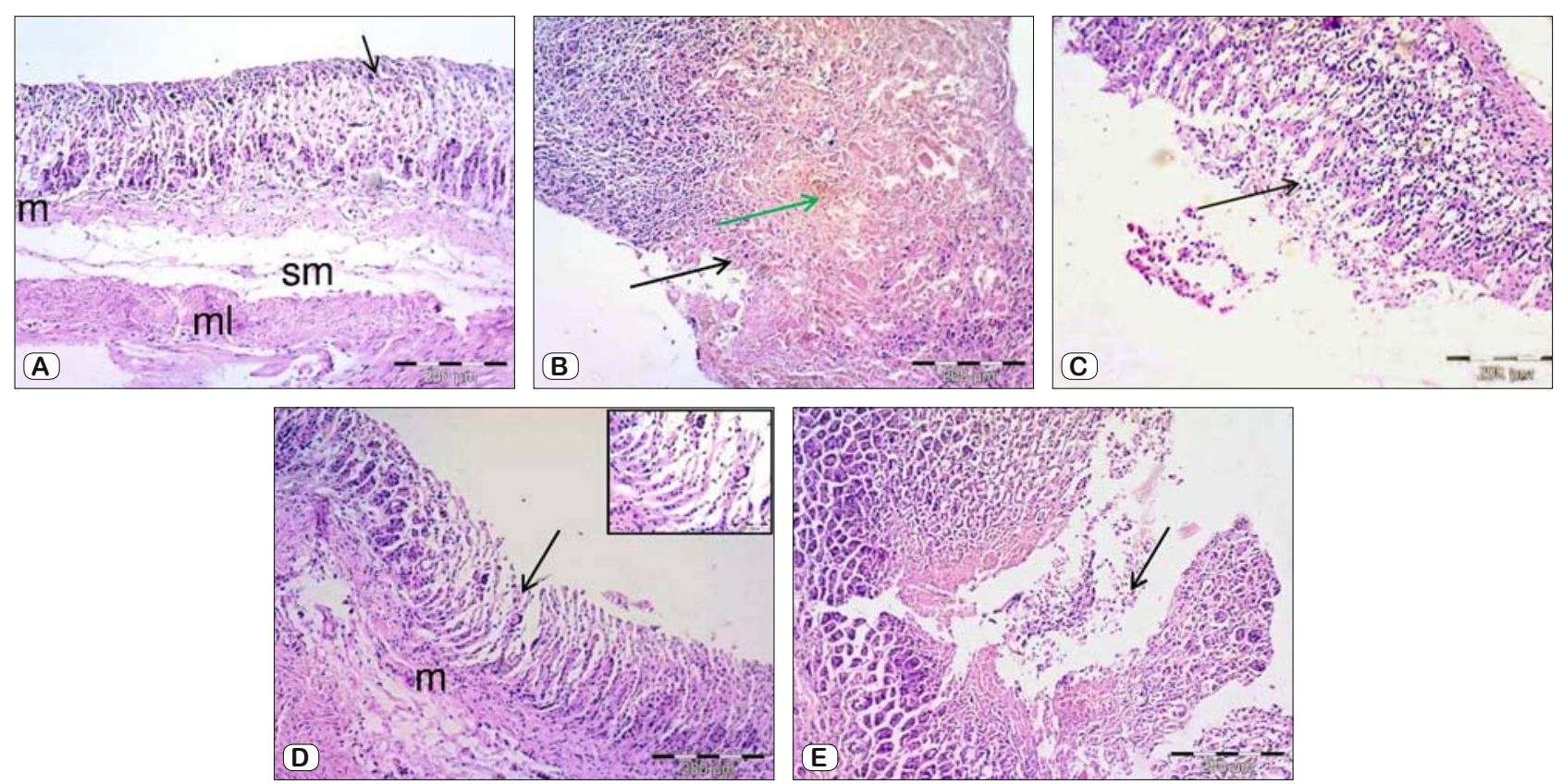

Fig. 4. A) A photomicrograph of control group gastric fundic mucosa showing gastric pits ( $\uparrow$ ) and long straight tubular fundic glands. Muscularis mucosa (m), Submucosa (sm), and muscularis externa (MI). B) A photomicrograph of CRS group showing large ulcerated area filled with necrotic tissue (black arrow) spanding the whole thickness. Notice areas of hemorrhage (green arrow). C) A photomicrograph Stressed Ang-(1-7) group showing erosion of the superficial part of the gastric mucosa and exfoliated cells (arrow). D) A photomicrograph of Stressed L-NNA-Ang-(1-7) group showing distortion of the glands with exfoliation of epithelial cell and ulceration of the surface mucosa (arrow) extending slightly into the muscularis mucosa $(\mathrm{m})$. Inset higher magnification showing degenerated and detached from their basement membrane x400. E) A photomicrograph of Stressed Indo-Ang-(1-7) treated showing large ulcerated area of fundic mucosa with exfoliation of epithelial cell (arrow). H\&EX100
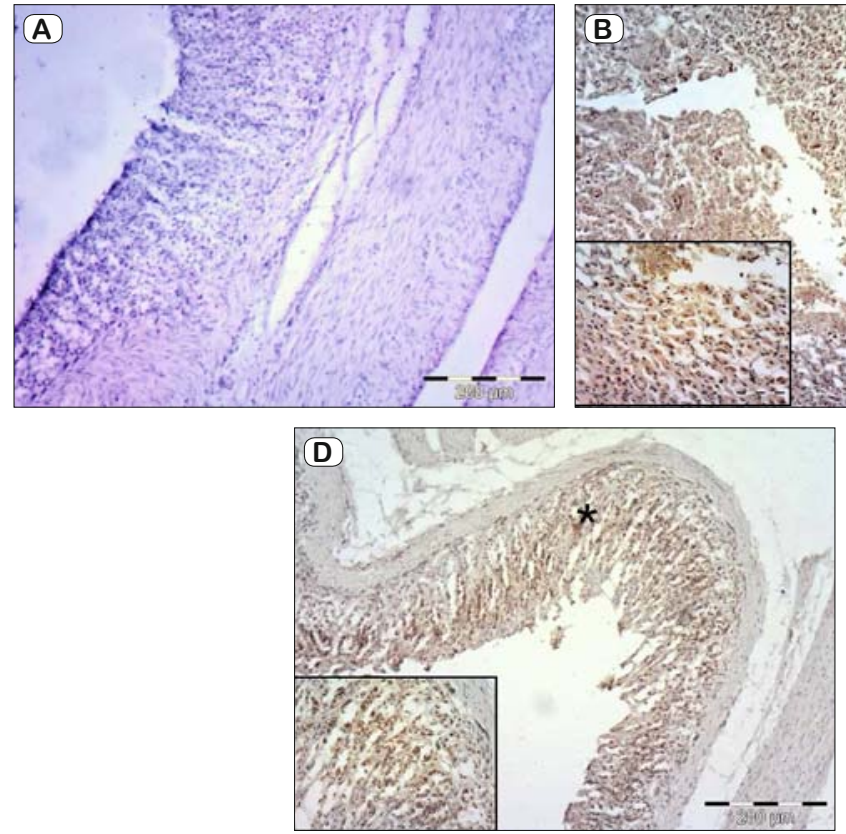
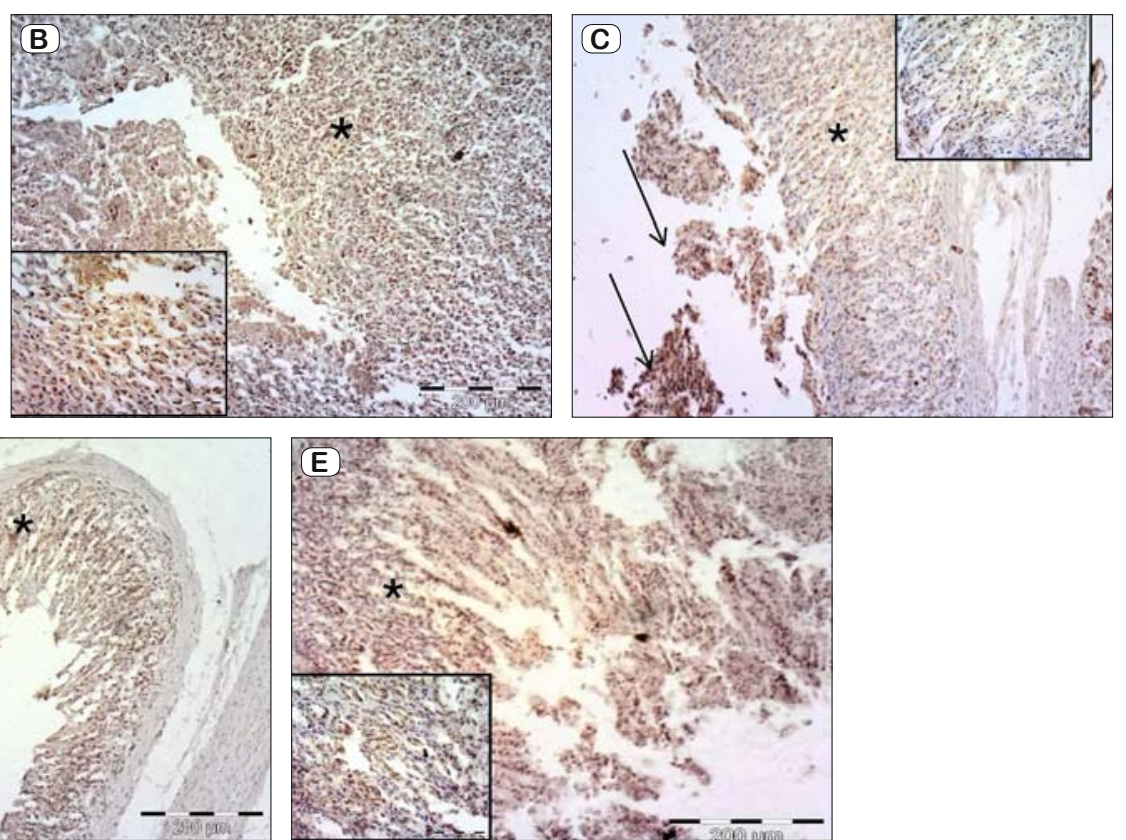

Fig. 5. A Photomicrograph of the fundic gland of immunostained for caspase 3 of A) The control group gastric fundic mucosa showing negative expression B) CRS group showing high positive expression in the ulcerated area. Notice the immunopositive cells shrunken and separated from the basement membrane (inset) C) Stressed Ang-(1-7) group showing mild expression among the fundic cells (*) and high expression in the exfoliated cells (arrows). Inset showing higher magnification of the immunopositive cells. D) Stressed L-NNA-Ang-(1-7) group showing moderate expression in the fundic epithelial cells. Inset showing higher magnification of the immunopositive cells E) Stressed Indo-Ang-(1-7) treated group showing high positive expression in the ulcerated area. Inset showing higher magnification of the immunopositive cells. $x 100$ Insets $x 400$ 
activity with higher gastric volume and $\mathrm{pH}$ as compared to nontreated CRS group (Tab. 1). At the same time, pre-treatment CRS group with Ang-(1-7) significantly lowered the gastric mucosal lipid peroxidase level, but with higher levels of $\mathrm{NO}$ and $\mathrm{PGE}_{2}$ as compared to non-treated CRS group (Figs 1, 2, 3).

- Co-administration of L-NNA or indomethacin with Ang-(1-7) before exposure to CRS significantly increased the gastric mucosal ulceration and increased the ulcer index to 4.21 and 4.66 as compared to the stressed Ang-(1-7) group and preventive index declined to $66.3 \%$ and $63.3 \%$, respectively (Tab. 2). In addition, these co-administrations significantly lowered the gastric juice volume, $\mathrm{pH}$, gastric mucosal $\mathrm{NO}$ and $\mathrm{PGE}_{2}$ levels as compared to the control and to the stressed Ang-(1-7) groups, but they were still significantly higher than in the non-treated CRS as shown in Table 1 and Figures 2 and 3. Moreover, there were significantly higher levels in the gastric juice free and total acidity, pepsin concentration and the gastric mucosal lipid peroxidase as compared to the control and stressed Ang-(1-7) groups, but there were still significantly lower than the nontreated CRS group (Tab. 1, Fig. 1).

- Examinations of sections of the fundus of stomach of control rats showed that the wall of the fundus of the stomach was formed of 4 classic layers namely, mucosa, submucosa, musculosa and serosa. The mucosa was occupied by simple branched tubular fundic gland (Fig. 4A). In the CRS group, large ulcerated area filled with necrotic tissue and areas of hemorrhage appeared spanning the whole thickness of the fundic wall (Fig. 4B). Small eroded areas of the superficial area of the fundic mucosa with exfoliation of some epithelial cells were observed in Stressed Ang-(1-7) (Fig. 4C), while in the Stressed L-NNA-Ang-(1-7) treated group the eroded area extend to the muscularis mucosa (Fig. 4D). In the Stressed Indo-Ang-(1-7) treated group, large ulcerated area of fundic mucosa with exfoliation of epithelial cell was noticed expanding the mucosa (Fig. 4E).

- Immunohistochemical results for caspase revealed a negative caspase 3 reaction in the epithelial cells of the fundic gland of the control group (Fig. 5A). Very strong caspase 3 positive reaction was observed in the cytoplasm of most of the fundic epithelial cells of the CRS group (Fig. 5B). In the Stressed Ang(1-7) group, few epithelial cells of the fundic gland showed faint reaction while strong reaction was noticed in the exfoliated cells (Fig. 5C). Moderate reaction was noticed in the cytoplasm of the fundic epithelial cells of the Stressed L-NNA-Ang-(1-7) treated group (Fig. 5D). Still strong reaction was observed in the eroded area of the Stressed Indo-Ang-(1-7) treated group (Fig. 5 E).

\section{Discussion}

Stress is a common contributor to development of peptic ulcer in humans. CRS is frequently used and clinically relevant experimental model for induction of acute gastric ulcer (5). The present study was an attempt to evaluate the possible gastro-protective effect of the Ang-(1-7). Since the discovery of Ang-(1-7) in 1976, the presence of this hectapeptide has been detected in brain, blood vessels, heart, kidney, liver, and stomach. Ang-(1-7) acting via its own G protein-coupled receptor (GPCR) called Mas Receptor exhibit the vasodilatory, antihypertensive and cardio-protective (1).

The data of the present study clearly demonstrated that CRS induced evident ulcers, which are in accordance with the observations of several researchers $(21,4)$. This occurs by enhancing the aggressive factors with severe extensive gastric damage as shown in our histological examination that can be explained by many causes. First, CRS in the present study significantly increased the gastric acidity, proteolytic activity with concomitant reduction in the volume of gastric juice as compared to control group, which is likely to be due to increase in the sympatho-adrenal activity, which occurs during stress (22).

Second, CRS significantly increased the gastric mucosal lipid peroxides level, as compared to the control group, which is in agreement with other investigators (21). This increase in the lipid peroxidation is a result of the elevation in the oxidative stress and ROS induced by stress. In addition, the reduction in gastric mucosal blood (GMB) flow occurring with stress increases malondialdehyde (MDA) level (23).

Moreover, the significant reduction of gastric mucosal NO and $\mathrm{PGE}_{2}$ levels in CRS rats can contribute to reduced mucosal blood flow by the vasoconstriction response, which leads to increase the production of free radicals, enhanced lipid peroxidation, impairment of anti-oxidizing enzyme activity and the increased generation of pro-inflammatory cytokines, such as TNF- $\alpha$ (24).

In the present study, pre-treatment with Ang-(1-7) significantly reduced the gastric ulcer index, gastric mucosal MDA level with concomitant increase in gastric mucosal concentrations of $\mathrm{PGE}_{2}$ and $\mathrm{NO}$ in comparison with the CRS group. In addition, there was an improvement in the severe gastric damages that were observed by the histological examination. The protective effect of Ang-(1-7) in our study against ulcer development might be due to its effect on gastric juice acid concentration and proteolytic activity. The inhibitory effect of Ang-(1-7) on the gastric acidity was suggested to be due to stimulation of gastric Mas receptor that lead to inhibition of $\mathrm{H}^{+}, \mathrm{K}^{+}$ATPase activities and subsequent decreased acid secretion and increased gastric $\mathrm{pH}$ (25).

Another explanation of the protective effect of Ang-(1-7) pretreatment may be due to its anti-oxidative effect as evidenced by decreasing the gastric mucosal lipid peroxides level, which is in accordance with the previous study of Liao et al (26). This effect can be also suggested to be due to stimulation of the gastric Mas receptor that leads to increased superoxide dismutase (SOD) activity, one of the most significant antioxidant systems functioning as a ROS scavenger. Another possible cause of antioxidant effect of Ang-(1-7) is increased NO/NOS ratio by stimulating NOS and increasing NO release by gastric Mas receptor stimulation (25) as observed in our study. Szlachcic et al (27) reported that Ang-(1-7) markedly decreased the expression and release of pro-inflammatory cytokines IL-1b and TNF-a suggesting that the anti-inflammatory properties of Ang-(1-7) may contribute to its protective activity.

Nitric oxide is a well-established mediator in the gastric mucosal defence and repair (28). NO modulates several elements of the gastric mucosal defence, including blood flow, neutrophil ad- 
hesion and mucus secretion. The effect of NO is, at least partially, mediated by an increase in cGMP content, and cGMP is normally broken down rapidly by phosphodiesterase-type 5 (6).

In the present study, the role of NO in the gastroprotective activity of Ang-(1-7) was evaluated by using L-NNA, NOS inhibitor. L-NNA pre-treatment significantly decreased the gastroprotective activity of Ang-(1-7) against CRS, evident in our measured parameters and histological examination. L-NNA pre-treatment inhibited the restoration of stress-induced decrease in NO level by Ang-(1-7) pre-treatment. Since NO is the endothelium derived relaxing factor, reduction of its level might contribute to reduced mucosal blood flow by the vasoconstriction response with a subsequent gastric damage (29).

In addition, L-NNA pre-treatment significantly decreased the gastric mucosal $\mathrm{PGE}_{2}$ as compared to Ang. (1-7) treated group. This effect can be explained by reduction of NO synthesis by L-NNA, which leads to attenuation of COX with subsequent decreased PGs synthesis, and it is possible to accept that NO might regulate the release and/ or the biosynthesis of $\mathrm{PGE}_{2}$ in the stomach after damage (24). Based on these findings, increased production of NO could be a potential target for the gastroprotective effect of Ang-(1-7) in this study.

Endogenous PGs are involved in the maintenance of mucosal integrity and blood flow. PGs also protect mucosa against potentially noxious agents. Moreover, PGs seem to stimulate the secretion of bicarbonate and mucus, inhibit the acid secretion, as well as regulate the mucosal cell turnover and repair in the stomach mucosa. Gastric mucosa of ulcer patients tends to generate smaller amounts of PGs of E and I series. This suggests that the insufficient production of protective PGs may play a primary role in the pathogenesis of gastric ulcer (23).

Therefore, in order to investigate the role of PGs in the gastroprotection offered by Ang-(1-7), rats were pre-treated with indomethacin, non-selective cyclooxygenase (COX) inhibitor, with Ang-(1-7) and then subjected to CRS. The results showed that pretreatment with indomethacin attenuated the protection afforded by Ang-(1-7) against CRS as evidenced by our biochemical and histological parameters. In addition, the result of the present work showed a marked reduction in NO level in the indomethacin pretreated group. This is attributed to the ability of indomethacin to up-regulate the endothelin-1 that leads to decrease production of gastric mucosal NO (30).

The involvement of PG in the anti-ulcer activity of Ang-(1-7) was further confirmed by direct measurement of the gastric $\mathrm{PGE}_{2}$ level in control, CRS and Ang-(1-7) pre-treated CRS groups. The results showed that CRS decreased the $\mathrm{PGE}_{2}$ level in gastric mucosa to that of control, whereas Ang-(1-7) repeated pre-treatment increased the $\mathrm{PGE}_{2}$ level and this effect can be explained by stimulation of Mas receptor by Ang-(1-7), which leads to activation of PG/COX pathway (31).

The maintenance of gastric mucosal integrity depends on the interplay between epithelial cell proliferation and apoptosis. Apoptosis has recently been suggested as a causative factor for cold restrained stress-induced gastric ulcer. Caspase-3, a key mediator for execution of apoptosis [programmed cell death], is involved in apoptotic cell death in stress-induced ulcer (32). As shown in our immunological examination, the cold restrained stress resulted in a dramatic activation of caspase-3, while the expression of cleaved caspase-3 was markedly decreased by the Ang-(1-7) pre-treatment by inhibiting the mitogen-activated protein kinase (MAPK)/NF- $\kappa$ B pathway (33). On the contrary, Ang-(1-7) has also been reported to induce apoptosis in circulating fibrocytes and human lung cancer cells. Thus, the anti-apoptotic effect of Ang-(1-7) is dependent on the cell type (34).

Previous studies have shown that NO either endogenously produced or exogenously applied in physiologically relevant concentrations acts as an endothelial cell survival factor, so NO signalling pathway largely contributes to the anti-apoptotic effect of Ang (1-7) (35) as evidenced in our study by increased caspase 3 expression in L-NNA and indomethacin pre-treatment groups.

In conclusion, current results demonstrated that the pathogenesis of gastric ulcer appears to be multifactorial depending on protective and aggressive factors. The gastroprotective effect of Ang. (1-7) was consistently accompanied by increased gastric mucosal blood flow, reduction in all acid parameters, and reduction in lipid peroxides. The mechanisms of Ang. (1-7) induced protection against ulceration were mediated by activation of $\mathrm{NO} / \mathrm{NOS}$ and $\mathrm{PG} /$ COX systems that were proved in our study by administrating LNNA (non-selective nitric oxide inhibitor) or indomethacin (COX inhibitor) before Ang. (1-7). Another explanation of the protective effect of Ang. (1-7) may be due to the anti-oxidant effect against induced oxidative stress, which is evidenced by marked attenuation in lipid peroxides level and also its anti- apoptotic effect.

Despite providing the effects of Ang-(1-7) therapy against ulcer development, this study has the limitation that the focus on the functional data and inflammatory markers did not include a mechanistic investigation into Ang-(1-7) mechanism of action in providing gastric protection. This will open the door for further future studies to prove its mechanisms in action. In addition, further studies in experimental animals and humans are warranted to further determine the therapeutic efficacy of Ang-(1-7) in various gastrointestinal disorders.

\section{References}

1. Pawlik M, Kwiecien S, Pajdo R, Ptak-Belowska A, Brzozowski B, Krzysiek-Maczka G, Strzalka M, Konturek S, Brzozowski T. Esophagoprotective activity of angiotensin-(1-7) in experimental model of acute reflux esophagitis. Evidence for the role of nitric oxide, sensory nerves, hypoxia-inducible factor-1alpha and proinflammatory cytokines. J Physiol Pharmacol 2014; 65 (6): 809-822.

2. Magierowski M, Jasnos K, Pawlik M, Krzysiek-Maczka G, PtakBelowska A, Olszanecki R, Kwiecien S, Korbut R, Brzozowski T. Role of angiotensin-(1-7) in gastroprotection against stress-induced ulcerogenesis. The involvement of mas receptor, nitric oxide, prostaglandins, and sensory neuropeptides. J Pharmacol Exp Ther 2013; 347 (3): 717-726.

3. Santos R. Angiotensin-(1-7). Hypertension 2014; 63 (6): 1138-1147.

4. Sachan N, Chandra P, Pal D. Assessment of Gastroprotective Potential of Delonix regia (Boj Ex Hook) Raf against Ethanol and Cold Restrain Stress-Induced Ulcer in Rats. Tropical J Pharm Res 2015; 14 (6): 1063-1070. 
5. Adinortey M, Ansah C, Galyuon I, Nyarko A. In vivo models used for evaluation of potential antigastroduodenal ulcer agents. Ulcers 2013; 2013 (12): 796405.

6. Kochar N, Chandewal A, Bakal R, Kochar P. Nitric Oxide and the Gastrointestinal Tract. Int J Pharm 2011; 7: 31-39.

7. Sánchez-Mendoza1 M, Cruz-Antonio L, Cupido-Sánchez1 M, GarcíaCastillo G, Arrieta J. Gastroprotective activity of caryophyllene oxide: the role of nitric oxide, prostaglandins and sulfhydryls. J Applied Pharmacol Sci 2014; 4 (09): 001-005.

8. Bregonzio C, Armando I, Ando H, Jezova M, Baiardi G, Saavedra J. Anti-inflammatory effects of angiotensin II AT1 receptor antagonism prevent stress-induced gastric injury. Am J Physiol Gastrointest Liver Physiol 2003; 285 (2): 414-423.

9. Alumets J, Kelund M, Hakanson R, Hedenbro J, Rehfeld J, Sundler F, Vallgren S. Gastric acid response to pyloric ligation in rats: is gastrin or histamine involved? J Physiol 1982; 323: 145-156.

10. Dronjak S, Gavrilovi L, Filipovi D, Radoj I. Immobilization and cold stress affect sympatho-adrenomedullary system and pituitary-adrenocortical axis of rats exposed to long-term isolation and crowding. Physiol Behav 2004; 81 (3): 409-415.

11. Satoh H, Amagase K, Ebara S, Akiba Y, Takeuchi K. Cyclooxygenase (COX)-1 and COX-2 both play an important role in the protection of the duodenal mucosa in cats. J Pharmacol Exp Ther 2013; 344: 189-195.

12. Pinheiro S, Simoes E, Silva A, Sampaio W, De Paula R, Mendes E, Bontempo E, Pesquero J, Walther T, Alenina N, Bader M. Nonpeptide AVE 0991 is an angiotensin-(1-7) receptor Mas agonist in the mouse kidney. Hypertension 2004; 44: 490-496.

13. Castro C, Santos R, Ferreira A, Bader M, Alenina N, Almeida A. Evidence for a functional interaction of the angiotensin-(1-7) receptor Mas with AT1 and AT2 receptors in the mouse heart. Hypertension 2005; 46: 937-942.

14. Robert A, Nezamis J, Phillips J. Effect of prostaglandin E1 on gastric secretion and ulcer formation in the rat. Gastroenterol 1968; 55 (4): 461-487.

15. Hano J, Bugajshi J, Danek L. The effect of neuroleptics on the development of gastric ulcer in rats exposed to restraint cold stress. Pol J Pharmacol Pharm 1976; 28 (1): 37-47.

16. Hara N, Hara Y, Natsume Y, Goto Y. Gastric hyperacidity and mucosal damage caused by hypothermia correlate with increase in GABA concentrations of the rat brain. Eur J Pharmacol 1991; 194 (1): 77-81.

17. Pratha V, Hogan D, Lane J, Williams P, Burton M, Lynn R, Karlstadt R. Inhibition of Pentagastrin-Stimulated Gastric Acid Secretion by Pantoprazole and Omeprazole in Healthy Adults. Dig Dis Sci 2006; 51 (1): 123-131.

18. Blandizzi C, Colucci R, Carignani D, Natale G, Lazzeri G, Crema F, Del Tacca M. Role of peripheral GABAB receptors in the regulation of pepsinogen secretion in anaesthetized rats. Eur J Pharmacol 1995; 294 (1): 191-200.

19. Bancroft J, Garble M. Theory and Practice of Histological Techniques. 5th edition, Churchill Livingstone: Harcourt, 2007; 85-98: 310314

20. Côté A, Silva R, Cuello A. Current protocols for light microscopy immunocytochemistry. In: Cuello AC, editor. Immunohistochemistry II: John Wiley \& Sons, 1993; 147-168, Chichester.
21. Dekanski D, Ristić S, Radonjić N, Petronijević N, Dekanski A, Mitrović D. Olive leaf extract modulates cold restraint stress-induced oxidative changes in rat liver. J Serb Chem Soc 2011; 76 (9): 1207-1218.

22. Fahami N, Ibrahim I, Kamisah Y, Ismail N. Palm vitamin E reduces catecholamines, xanthine oxidase activity and gastric lesion in rats exposed to water immersion restraint stress. BMC Gastroenterol 2012; 12: 54.

23. Saxena B, Singh S. Investigations on gastroprotective effect of citalopram, an antidepressant drug against stress and pyloric ligation induced ulcers. Pharmacol Rep 2011; 63 (6): 1413-1426.

24. Nur Azlina M, Kamisah Y, Chua K, Ibrahim I, Qodriyah H. Preventive Effects of Tocotrienol on Stress-induced Gastric Mucosal Lesions and Its Relation to Oxidative and Inflammatory Biomarkers. PLoS One 2015; 10 (10): e0139348.

25. Zimmerman M. Angiotensin II and angiotensin-1-7 redox signaling in the central nervous system. Curr Opin Pharmacol 2011; 11 (2): 138-143.

26. Liao X, Wang L, Yang C, He J, Wang X, Guo R, Lan A, Dong X, Yang Z, Wang H. Cyclooxygenase mediates cardioprotection ofangiotensin-(1-7) against ischemia/reperfusion-induced injury through the inhibition of oxidative stress. Mol Med Rep 2011; 4: 1145-1150.

27. Szlachcic A, Krzysiek-Maczka G, Pajdo R, Targosz A, Magierowski M, Jasnos K, Drozdowicz D, Kwiecien S, Brzozowski T. The impact of asymmetric dimethylarginine (ADAMA), the endogenous nitric oxide (NO) synthase inhibitor, to the pathogenesis of gastric mucosal damage. Curr Pharm Des 2013; 19: 90-97.

28. Konturek P, Brzozowski T, Burnat G, Szlachcic A, Koziel J, Kwiecien S, Konturek S, Harsch I. Gastric ulcer healing and stresslesion preventive properties of pioglitazone are attenuated in diabetic rats. J. Physiol. Pharmacol 2010; 61: 429-436.

29. Magierowski M, Magierowska K, Kwiecien S, Brzozowski T. Gaseous mediators nitric oxide and hydrogen sulphide in the mechanism of gastrointestinal integrity, protection and ulcer healing. Molecules 2015; 20 (5): 9099-9123.

30. Prabha P, Karpagam T, Varalakshmi B, Packiavathy A. Indigenous anti-ulcer activity of Musa sapientum on peptic ulcer. Pharmacognosy Res 2011; 3 (4): 232-238.

31. Pawlik M, Pajdo R, Kwiecien S, Ptak-Belowska A, Sliwowski Z, Mazurkiewicz-Janik M, Konturek S, Pawlik W, Brzozowski T. Nitric oxide (NO)-releasing aspirin exhibits a potent esophagoprotection in experimental model of acute reflux esophagitis. Role of nitric oxide and proinflammatory cytokines. J Physiol Pharmacol 2012; 62: 75-86.

32. Liu X, Chen Z, Mao N, Xie Y. The protective of hydrogen on stressinduced gastric ulceration. Int Immunopharmacol 2012; 13 (2): 197-203.

33. Meng Y, Yu C, Li W, Li T, Luo W, Huang S, Wu P, Cai S, Li X. Angiotensin-converting enzyme 2/angiotensin-(1-7)/Mas axis protects against lung fibrosis by inhibiting the MAPK/NF- $\kappa$ B pathway. Am J Respir Cell Mol Biol 2014; 50 (4): 723-736.

34. Yang H, Bian Y, Zhang H, Gao F, Xiao C, Liang B, Li J, Zhang N, Yang Z. Angiotensin-(1-7) treatment ameliorates angiotensin II-induced apoptosis of human umbilical vein endothelial cells. Clin Exp Pharmacol Physiol 2012; 39 (12): 1004-1010.

35. Passos-Silva D, Verano-Braga T, Santos R. Angiotensin-(1-7): beyond the cardio-renal actions. Clin Sci (Lond) 2013; 124 (7): 443-456.

Received August 13, 2016. Accepted August 30, 2016. 